\title{
Unambiguous Acquisition and Tracking Technique for General BOC Signals
}

\author{
Feng SHEN ${ }^{1}$, Guanghui XU ${ }^{1}$, Joon Wayn CHEONG $^{2}$, Haiyu FENG ${ }^{1}$ \\ ${ }^{1}$ College of Automation, Harbin Engineering University, Street Nantong, Harbin, China \\ ${ }^{2}$ School of Electrical Engineering and telecommunications, University of New South Wales, Sydney, NSW, 2052, Australia
}

\{sf407, xghdh407, fhydh407\}@126.com, cjwayn@unsw.edu.au

\begin{abstract}
This article presents a new unambiguous acquisition and tracking technique for general Binary Offset Carrier (BOC) ranging signals, which will be used in modern GPS, European Galileo system and Chinese BeiDou system. The test criterion employed in this technique is based on a synthesized correlation function which completely removes positive side peaks while keeping the sharp main peak. Simulation results indicate that the proposed technique completely removes the ambiguity threat in the acquisition process while maintaining relatively higher acquisition performance for low order BOC signals. The potential false lock points in the tracking phase for any order BOC signals are avoided by using the proposed method. Impacts of thermal noise and multipath on the proposed technique are investigated; the simulation results show that the new method allows the removal of false lock points with slightly degraded tracking performance. In addition, this method is convenient to implement via logic circuits.
\end{abstract}

\section{Keywords}

Modern GPS, Galileo, BeiDou, BOC, unambiguous acquisition and tracking, multipath, thermal noise.

\section{Introduction}

In recent years, applications of Global Navigation Satellite System (GNSS) are developing rapidly and attracting a great interest. However, GNSSs have to coexist in a limited number of frequency bands [1]. The traditional GPS L1 signal uses a spread spectrum technique with a Binary-Phase Shift Keying (BPSK) modulation. With the upcoming GNSS generation some civil signals will implement BOC modulation that allows improved code delay tracking while offering a spectral separation from BPSK signals due to its split spectrum [2]. However, the main drawback of every BOC modulated signal is its multi-peak AutoCorrelation Function (ACF) that implies possible false acquisition or biased tracking which will result in biased code delay measurements of up to hundreds of meters if no special care is taken.
Several techniques have been investigated to remove this biased threat [3]-[16]. Sideband filtering techniques in [3]-[5] can mitigate the ambiguity threat completely, but the filtering and the dual sideband processing increases the implementation complexity and causes correlation loss due to the mismatch between the received code (BOC) and the local code (shifted BPSK). Besides, these methods produce a wider ACF peak than the main ACF peak of a sine-phased BOC if a classical delay lock loop is used in signal tracking. Therefore, an additional transition from acquisition to tracking is necessary to avoid falsely locking onto an ACF sidepeak. [6], [7] are autocorrelation function based acquisition and tracking techniques. Bump-Jumping (BJ) technique aims at determining whether or not the peak being tracked is the correct one [7]. It exhibits high tracking accuracy when it is locked on the main peak, but the acquisition may have a high probability of false alarm when the signal-to-noise ratio is low. In addition, it needs time to recover from false lock, which makes it inapplicable in some time critical applications. Sub-carrier processing method such as Double Estimator Technique in [8] can completely solve the ambiguous problem while maintaining the tracking accuracy, but this method needs additional tracking loop for Sub-carrier. Sidepeak cancellation techniques [9]-[16] are based on the combination of constitutive sub-correlations of the BOC autocorrelation function. A General Removing Ambiguity via Side peak Suppression (GRASS) technique is proposed in [9] for sine-BOC $(k n, n)$ signal and in [10] for cosine-BOC $(k n, n)$ signal, which can suppress all of the undesired side peaks by combining the cross-correlation function between the received signal and the local auxiliary signal with the ACF of the received signal. However, the method in [10] presents high implementation complexity because of its residual two positive side peaks, which need to be calculated previously under different frond-end bandwidth. A Pseudo-CorrelationFunction (PCF)-based method presented in [12] does not have any side peaks and can completely remove all of the false lock points on the discriminator output. However, it is only work with sine phased BOC signals. A method referred to Autocorrelation Side-Peak Cancellation Technique (ASPeCT) was proposed in [16] which can realize both unambiguous acquisition and tracking. However, it is only dedicated to sine-BOC $(n, n)$ signal. As a summation of the methods mentioned above, most of the techniques can not be 
used for both BOC signals acquisition and tracking process simultaneously, and most of them are also only suitable to a dedicated kind of BOC modulated signal.

In this paper, an ambiguity removal technique suitable for generic $\operatorname{BOC}(x, y)$ signals is developed. Different from aforementioned side-peak cancellation techniques, the proposed method can realize both unambiguous acquisition and tracking process for generic $\mathrm{BOC}(x, y)$ signals. By employing two symmetrical local auxiliary signals, and combining the first correlation function between the received signal and one local auxiliary signal with the second correlation function between the received signal and another local auxiliary signal, the proposed technique constructs a test criterion which removes all of the undesired positive side peaks. Meanwhile, the false lock points can also be avoided by using modified Early-Minus- Late Power discriminator.

The remainder of this paper is organized as follows. Firstly, we discuss the generic solution of the local modulated symbols for both sine-phased and cosine-phased BOC modulations. Then, the acquisition performance including detection probability of the proposed method is analyzed and evaluated. Meanwhile, the tracking accuracy including multipath and thermal noise resistance performance are discussed in detail. Followed by the implementation complexity analysis and structure simplification. Finally, the contributions of this paper and future steps are summarized.

\section{BOC Modulated Signals and Method Description}

\subsection{BOC Signal Model Definition}

In this paper, a BOC modulation is denoted as $\operatorname{BOC}(x, y)$, where $x$ means the ratio of the square wave frequency $f_{s}$ to $1.023 \mathrm{MHz}$, and $y$ denotes the ratio of the spreading code rate $f_{c}$ to $1.023 \mathrm{MHz}$. The ratio $M=2 x / y$ is referred as BOC-modulation order and it is constrained to a positive integer value. Since $M$ is even in most of BOC signals employed in GNSS, for simplicity, we will discuss only the even case. The baseband direct sequence spread spectrum signal can be expressed as [12]

$$
S(t)=\sum_{i}(-1)^{c_{i}} p\left(t-i T_{c}\right)
$$

where $c_{i}$ is the spreading sequence of the binary digits $\{0,1\}$, $T_{c}$ is the symbol duration, and $p(t)$ is the modulated symbol which is further divided into $M$ segments with equal length $T_{s}=T_{c} / M$ for sine-BOC signal. Then the modulated symbol can be represented as

$$
p(t)=\sum_{k=0}^{M-1} d_{k} \psi_{k}(t)
$$

The shape vector can be described as $d_{k}=(-1)^{k}, k=$ $0,1, \ldots, M-1$; and the basic shape $\psi_{k}(t)$ can be expressed as

$$
\psi_{k}(t)= \begin{cases}1 & t \in\left[k T_{c} / M,(k+1) T_{c} / M\right] \\ 0 & \text { others. }\end{cases}
$$

For cosine-BOC signal, the modulated symbol is divided into $2 M$ segments with equal length $T_{s}=T_{c} / 2 M$, then

$$
p(t)=\sum_{k=0}^{2 M-1} d_{k} \psi_{k}(t)
$$

where $d_{k}=(-1)^{\lceil k / 2\rceil}, k=0,1, \ldots, 2 M-1,\lceil\cdot\rceil$ represents the ceiling operation; and

$$
\psi_{k}(t)= \begin{cases}1 & t \in\left[k T_{s},(k+1) T_{s}\right] \\ 0 & \text { others }\end{cases}
$$

Specially, for Multiplexed Binary Offset Carrier (MBOC) modulated signal $M=12$ which has been widely used in modern GNSS, $d_{k}=(-1)^{\lfloor k / 6\rfloor} \gamma_{1}+(-1)^{k} \gamma_{2}[13]$; where $\lfloor\cdot\rfloor$ represents the floor operator, $\gamma_{1}=\sqrt{10 / 11}$ and $\gamma_{2}=\sqrt{1 / 11}$ are the amplitude weighting factors. The basic shape $\psi_{k}(t)$ is the same as (3).

\subsection{Proposed Method Description}

In [12], Yao et al. presented the Cross-Correlation Function $(\mathrm{CCF})$ between the spreading signal and the Stepshape Code Symbol signal (SCS) which have the same $f_{c}$ and $M$. In fact, the step-shape modulated symbol $p(t)$ can be identified by vector $\mathbf{d}_{s c s}=\left[d_{0}, d_{1}, \ldots, d_{M-1}\right]^{T}$ and spreading sequence rate $f_{c}=1 / T_{c}$. So a step-shape modulated symbol can be denoted by $p\left(t, \mathbf{d}_{s c s}, f_{c}\right)$. Some examples of stepshape modulated symbols are shown in Fig. 1. In this paper, the CCF will be further generalized to cosine-BOC signals.
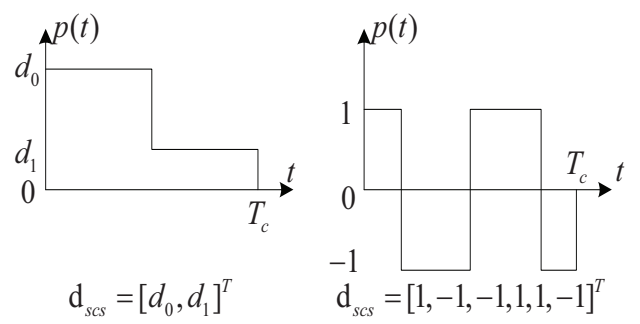

Fig. 1. Step-shape modulated symbols.

Under the assumption that $\left\{c_{i}\right\}$ have ideal autocorrelation characteristics, the CCF between cosine-BOC and SCS signals using their waveforms $p_{B O C_{c}}\left(t ; \mathbf{d}_{B O C_{C}} ; f_{C}\right)$ and $p_{S C S}\left(t ; \mathbf{d}_{S C S} ; f_{c}\right)\left(\mathbf{d}_{S C S}=\left[d_{0}, d_{1}, \ldots, d_{2 M-1}\right]^{T}\right)$ can be expressed as

$R_{B / L}(\tau)=\left\{\begin{array}{c}\left(\tau / T_{s}-k\right)\left(r_{k+1}-r_{k}\right)+r_{k} \\ \tau \in\left[k T_{s},(k+1) T_{s}\right], \\ \left(\tau / T_{s}-k+2 M\right)\left(r_{k-2 M+1}-r_{k-2 M}\right)+r_{k-2 M} \\ 0 \quad \tau \in\left[(k-2 M) T_{s},(k-2 M+1) T_{s}\right],\end{array}\right.$

where $k=0,1, \ldots, 2 M-1$, and $r_{k}$ is the Aperiodic CrossCorrelation Function (ACCF) between $\mathbf{d}_{B O C_{C}}$ and $\mathbf{d}_{S C S}$, i.e., 


$$
r_{k}\left(\mathbf{d}_{S C S}\right)=\left\{\begin{array}{lc}
\frac{1}{2 M} \sum_{i=0}^{2 M-1-k}(-1)^{\lceil i / 2\rceil} d_{i+k}, & 0 \leq k \leq 2 M-1, \\
\frac{1}{2 M} \sum_{i=0}^{2 M-1+k}(-1)^{\lceil(i-k) / 2\rceil} d_{i} \\
0 & 1-2 M \leq k<0, \\
0 & |k| \geq 2 M
\end{array}\right.
$$

From (6) and (7), it can be noticed that $R_{B / L}\left(\frac{k T_{c}}{2 M}\right)=r_{k}$ and that $R_{B / L}$ is piecewise linear between the values at $\frac{k T_{C}}{2 M}$. Therefore, the shape of $R_{B / L}$ can be changed by changing the shape vectors of those two local step-shape modulated symbols

The proposed method in this paper uses two local signals with step-shape modulated symbols which noncoherently combine the outputs of the correlators to create a no-side-peak function instead of the original ambiguous ACF which contains 2 or more side-peaks. The CCF between a cosine-BOC $(k n, n)$ signal and two local step-shape modulated signals will be calculated as following.

The modulated symbol of one local signal is denoted as $p_{S C S_{c}}\left(t, \mathbf{d}_{1}, f_{c}\right)$, where $\mathbf{d}_{1}=\left[d_{0}^{(1)}, d_{1}^{(1)}, \ldots, d_{2 M-1}^{(1)}\right]^{T}$, and the modulated symbol of another local signal, denoted as $p_{S C S_{c}}\left(t, \mathbf{d}_{2}, f_{c}\right)$, is the mirror image of $p_{S C S_{c}}\left(t, \mathbf{d}_{1}, f_{c}\right)$, i.e.,

$$
d_{k}^{(2)}=d_{2 M-k-1}^{(1)} \text {. }
$$

The correlation functions between the received cosine$\operatorname{BOC}(k n, n)$ signal and these two local signals can be denoted as $R_{B / L_{1}}(\tau)$ and $R_{B / L_{2}}(\tau)$, respectively. Note that $(-1)^{\lceil i / 2\rceil}=(-1)^{\lceil(2 M-i-1) / 2\rceil}, i=0,1, \ldots, 2 M-1$. Substituting it into (7) yields

$$
\begin{aligned}
r_{k}^{(2)} & =\frac{1}{2 M} \sum_{i=0}^{2 M-1-k}(-1)^{\lceil i / 2\rceil} d_{i+k}^{(2)} \\
& =\frac{1}{2 M} \sum_{i=0}^{2 M-1-k}(-1)^{\lceil(2 M-i-1) / 2\rceil} d_{2 M-k-i-1}^{(1)} \\
& =\frac{1}{2 M} \sum_{i=0}^{2 M-1-k}(-1)^{\lceil i / 2\rceil} d_{i-k}^{(1)} \\
& =r_{-k}^{(1)} .
\end{aligned}
$$

Substituting (9) into (6), we have

$$
R_{B / L_{2}}(\tau)=R_{B / L_{1}}(-\tau)
$$

Just as the case in [12], a generic solution (i.e. the specific shape vectors of those two local step-shape modulated symbols) for high order $\operatorname{BOC}(k n, n)$ signal is hard to be given. For simplicity, a specific family of modulated symbol of local signal is dedicated to thereinafter. For cosine-BOC $(k n, n)$ signal, the shape vectors of two local step-shape modulated symbols can be expressed as:

$$
\begin{aligned}
& d_{1}(k)=\left[\sqrt{\frac{2 M}{1+k^{2}}}, 0, \ldots, 0, k \sqrt{\frac{2 M}{1+k^{2}}}\right]^{T}, \\
& d_{2}(k)=\left[k \sqrt{\frac{2 M}{1+k^{2}}}, 0, \ldots, 0, \sqrt{\frac{2 M}{1+k^{2}}}\right]^{T} .
\end{aligned}
$$

Note that these two step-shape symbols have been energy normalized and the shapes are identified by the shape factor $k=d_{2 M-1}^{(1)} / d_{0}^{(1)}, k \in[0,1)$. After substituting (11) into (7), it is interesting to find that

$$
\left\{\begin{array}{l}
r_{0}^{(1)}=r_{0}^{(2)}, \\
r_{i}^{(1)} \cdot r_{i}^{(2)} \geq 0, \quad i \neq 0, \\
\left|r_{i}^{(1)}+r_{i}^{(2)}\right|=\left(\frac{1+k}{1-k}\right)\left|r_{i}^{(1)}-r_{i}^{(2)}\right|, \quad i \neq 0 .
\end{array}\right.
$$

As analyzed above, we can draw the following conclusions.

(1) The CCFs between received BOC signal and local SCS signals are piecewise linear, and they are symmetrical relative to the perpendicular axis.

(2) The CCFs have the same zero crossing points and the same sign when $|\tau|>T_{S}$ as shown in Fig. 2; and the ACCF $r_{k}^{(1)}, r_{k}^{(2)}$ follow the terms in (12).

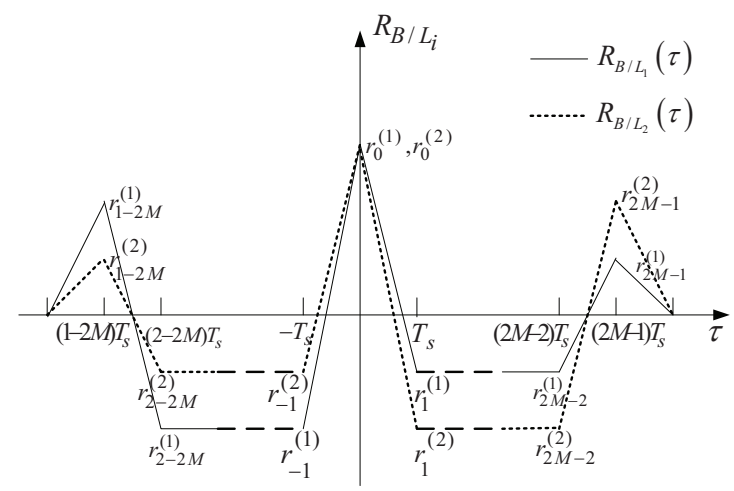

Fig. 2. Schematic diagram of correlation function $R_{B / L_{i}}(\tau)$.

Utilizing the characteristics of CCFs and the relationships shown in (10) and (12), it is possible to obtain a Synthesized Correlation Function (SCF) that completely removes any ACF side-peak. This eliminates the ambiguity threat in the acquisition and tracking process. Similar to [12], the SCF can be chosen as

$\bar{R}(\tau)=\left(R_{B / L_{1}}+R_{B / L_{2}}\right)^{2}-\left(\left(\frac{1+k}{1-k}\right) \cdot\left(R_{B / L_{1}}-R_{B / L_{2}}\right)\right)^{2}$.

In order to realize the acquisition and tracking process for both sine- and cosine-BOC $(k n, n)$ signals in the same loop without changing the receiver architecture, the SCF for sine-BOC $(k n, n)$ signal cannot be changed. Therefore, the modulated symbols of local signals for sine-BOC $(k n, n)$ signal are chosen as $p_{S C S_{S}}\left(t ; \mathbf{d}_{1} ; f_{c}\right)$ and $p_{S C S_{S}}\left(t ; \mathbf{d}_{2} ; f_{c}\right)$, wherein

$$
d_{k}^{(2)}=-d_{M-k-1}^{(1)} \text {. }
$$

Substituting (14) into the ACCF in [12], it can be seen that

$$
\begin{aligned}
r_{k}^{(2)} & =\frac{1}{M} \sum_{i=0}^{M-1}(-1)^{i} d_{i+k}^{(2)} \\
& =(-1)^{1-M+2 i} \frac{1}{M} \sum_{i=0}^{M-1}(-1)^{M-i-1}\left(-d_{M-i-k-1}^{(1)}\right) \\
& =\frac{1}{M} \sum_{i=0}^{M-1}(-1)^{i} d_{i-k}^{(1)} \\
& =r_{-k}^{(1)}
\end{aligned}
$$

Corresponding to cosine-BOC $(k n, n)$ signals, for sine$\operatorname{BOC}(k n, n)$ signal, we have $R_{B / L_{2}}(\tau)=R_{B / L_{1}}(-\tau)$; and the shape vectors of two local step-shape modulated symbols, similar with the shape vectors given in (11), are chosen as 


$$
\begin{aligned}
& d_{1}(k)=\left[\sqrt{\frac{M}{1+k^{2}}}, 0, \ldots, 0,-k \sqrt{\frac{M}{1+k^{2}}}\right]^{T}, \\
& d_{2}(k)=\left[k \sqrt{\frac{M}{1+k^{2}}}, 0, \ldots, 0,-\sqrt{\frac{M}{1+k^{2}}}\right]^{T} .
\end{aligned}
$$

Substituting (16) into the ACCF in [12], the same conclusions as (12) can be drawn for sine-BOC $(k n, n)$ signals. Therefore, the $\operatorname{SCF} \bar{R}(\tau)$ is also suitable for sine-BOC $(k n, n)$ signal when the shape vectors (16) are adopted for sinephased BOC signals.

Considering the fact that MBOC signal is the combination of two sine phased BOC signals, and most of the energy are located at sine-BOC $(1,1)$ signal, so that the ACF of the MBOC signal is mainly decided by sine-BOC $(1,1)$ signal. Therefore, the shape vectors of the two local step-shape modulated symbols are chosen like sine-BOC $(1,1)$ signal, i.e.,

$$
\begin{aligned}
d_{1}(k) & = \\
& {[\underbrace{\sqrt{\frac{2}{1+k^{2}}}, \ldots, \sqrt{\frac{2}{1+k^{2}}}}_{6}, \underbrace{-k \sqrt{\frac{2}{1+k^{2}}}, \ldots,-k \sqrt{\frac{2}{1+k^{2}}}}_{6}]^{T} }
\end{aligned}
$$$$
d_{2}(k)=
$$

$$
[\underbrace{k \sqrt{\frac{2}{1+k^{2}}}, \ldots, k \sqrt{\frac{2}{1+k^{2}}}}_{6}, \underbrace{-\sqrt{\frac{2}{1+k^{2}}}, \ldots,-\sqrt{\frac{2}{1+k^{2}}}}_{6}]^{T}
$$

Figures 3 to 5 show the SCFs of the proposed method and the impact of a front-end filter with a $6 \mathrm{MHz}$ doubleside bandwidth on the SCFs for MBOC, sine-BOC and cosine-BOC, respectively. As can be seen, the low-pass filter changes the shapes of the correlation functions $R_{B / L_{1}}$ and $R_{B / L_{2}}$. Note that the SCFs do not have any positive side peak. Therefore, the proposed method can completely remove the ambiguity threat for various BOC signals. Meanwhile, the main peak of the SCFs maintains its sharpness which translates into good tracking performance. Two negative side-peaks adjacent to the main peak are an artefact of unmatched slopes (refer to (12)) between the two correlation functions when $|\tau|<T_{s}$ as shown in Fig. 2. However, by using the special designed EMLP discriminator for the proposed method which will be illustrated in Section 4, these side-peaks will not maintain a tracking lock as they are negative valued [16].

\section{Acquisition using Proposed Method}

\subsection{Detection Probability Analysis.}

Figure 6 illustrates the receiver acquisition structure of the proposed method, wherein $(+/-)^{2}$ represents $\left(I_{1 p} \pm\right.$ $\left.I_{2 p}\right)^{2}$ or $\left(Q_{1 p} \pm Q_{2 p}\right)^{2}, I_{1 p}, I_{2 p}$ and $Q_{1 p}, Q_{2 p}$ are the in-phase and quadra-phase integrator outputs, respectively.

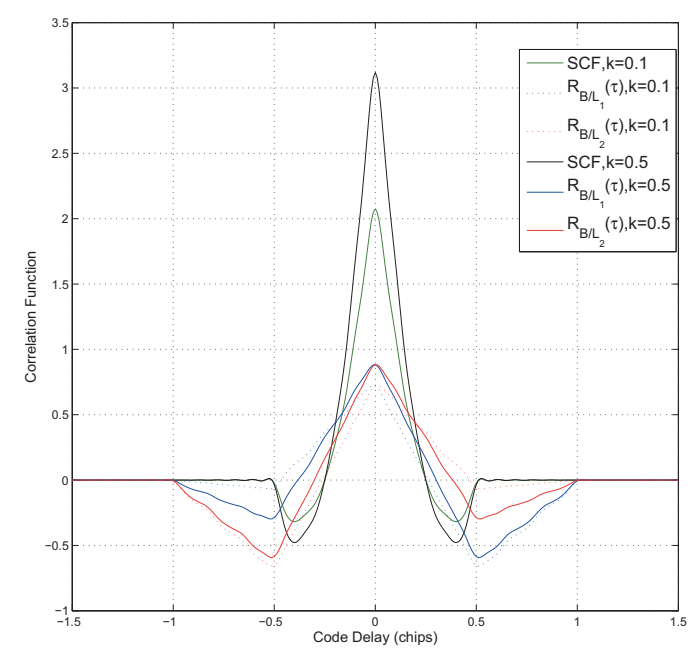

Fig. 3. $R_{B / L_{i}}$ and SCF for MBOC signal, $k=0.1$ and $k=0.5$, with $6 \mathrm{MHz}$ frond-end filter (double-sided).

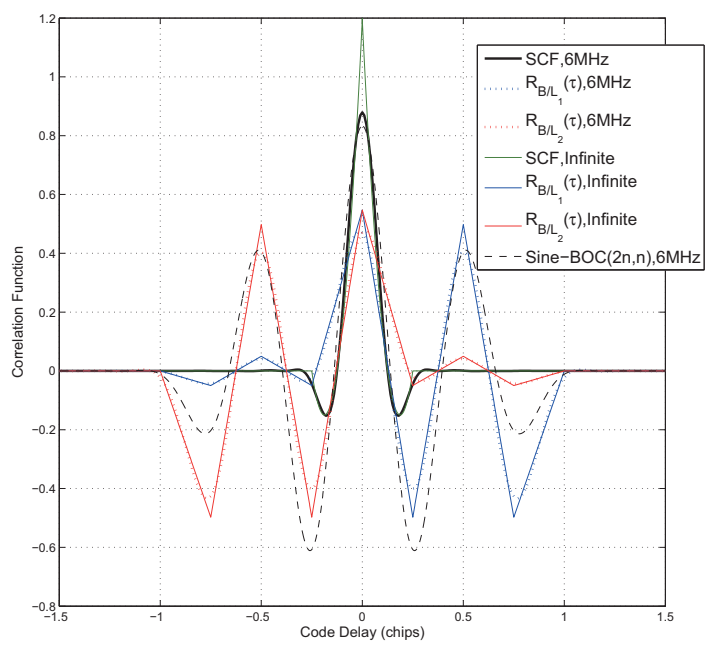

Fig. 4. $R_{B / L_{i}} \mathrm{SCF}$ and $\mathrm{ACF}$ for sine-BOC $(2,1)$ signal, $k=0.1$, with front-end filter $6 \mathrm{MHz}$ and infinite bandwidth.

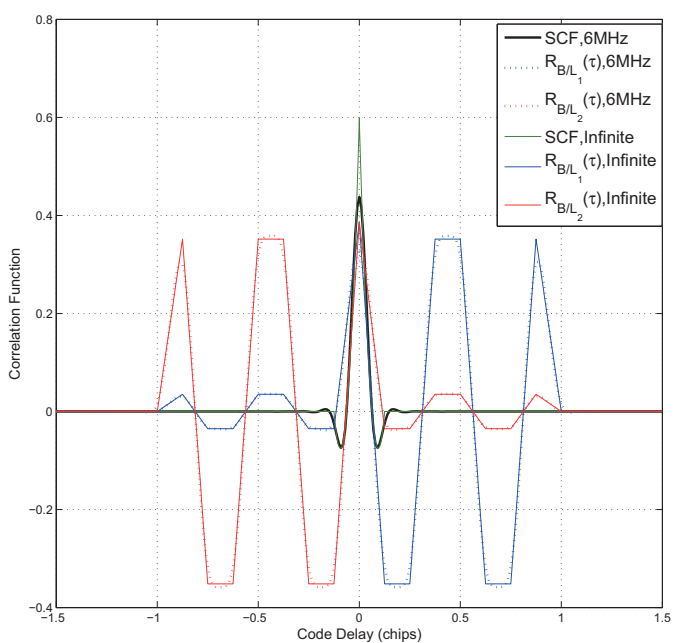

Fig. 5. $R_{B / L_{i}}$ and SCF for cosine-BOC $(2,1)$ signal, $k=0.1$, with front-end filter $6 \mathrm{MHz}$ and infinite bandwidth. 


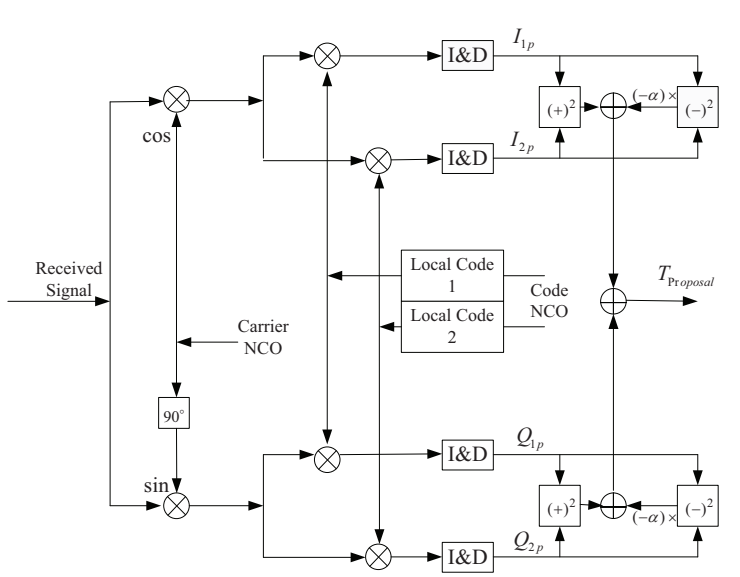

Fig. 6. Block diagram of the proposed acquisition structure.

Since the discriminator is non-coherent, we can ignore the data modulation. Therefore, the received Intermediate Frequency (IF) signal can be modeled as

$$
r(t)=A \exp \left(2 \pi f_{I F} t+\theta\right) s(t-\tau)+n(t)
$$

where $A$ is the signal amplitude, $f_{I F}$ is the intermediate frequency of the receiver, $\theta$ is the phase of the carrier, $\tau$ is the propagation code delay, $s(t)$ is the baseband direct sequence spread spectrum signal, $n(t)$ is the band-limited white noise with zero mean and double-sided power spectral density $N_{0}$, respectively.

As shown in Fig. 6, the test criterion of the proposed method can be expressed as

$T_{\text {proposal }}=\sum_{i=1}^{N}\left[\begin{array}{l}\left(\left(I_{B / L_{1}, i}+I_{B / L_{2}, i}\right)^{2}+\left(Q_{B / L_{1}, i}+Q_{B / L_{2}, i}\right)^{2}\right)- \\ \alpha\left(\left(I_{B / L_{1}, i}-I_{B / L_{2}, i}\right)^{2}+\left(Q_{B / L_{1}, i}-Q_{B / L_{2}, i}\right)^{2}\right)\end{array}\right]$

where $\alpha=\left(\frac{1+k}{1-k}\right)^{2}, k$ is the shape factor. $I_{B / L}$ and $Q_{B / L}$ are the in-phase and quadrature CCF results between the incoming BOC signal and the local auxiliary signal, respectively, which are expressed as

$$
\begin{aligned}
I_{B / L_{j}, i} & =(A / 2) \operatorname{sinc}\left(\pi \Delta f T_{c o h}\right) R_{B / L_{j}}(\Delta \tau) \cos (\Delta \varphi)+n_{I_{j}, i} \\
Q_{B / L_{j}, i} & =(A / 2) \operatorname{sinc}\left(\pi \Delta f T_{c o h}\right) R_{B / L_{j}}(\Delta \tau) \sin (\Delta \varphi)+n_{Q_{j}, i}
\end{aligned}
$$

where $j=1,2, \operatorname{sinc}(x)=\sin (x) / x . \quad T_{c o h}$ is the integration time. $\Delta f, \Delta \tau, \Delta \varphi$ refer the frequency wipe-off error, the code delay and carrier phase estimation errors, respectively. Since $n_{I_{j}, i}$ and $n_{Q_{j}, i}$ are generated by the same noise process, its probability density function (pdf) follows a Gaussian distribution [10]. Therefore, at a code delay of $\Delta \tau$, the joint distribution of the four branches is

$$
\left(I_{B / L_{1}}(\Delta \tau), I_{B / L_{2}}(\Delta \tau), Q_{B / L_{1}}(\Delta \tau), Q_{B / L_{2}}(\Delta \tau)\right) \sim N\left(\mu, \sigma^{2}\right)
$$

where

$$
\mu=(A / 2) \operatorname{sinc}\left(\pi \Delta f T_{c o h}\right)\left[\begin{array}{l}
R_{B / L_{1}}(\Delta \tau) \cos (\Delta \varphi) \\
R_{B / L_{2}}(\Delta \tau) \cos (\Delta \varphi) \\
R_{B / L_{1}}(\Delta \tau) \sin (\Delta \varphi) \\
R_{B / L_{2}}(\Delta \tau) \sin (\Delta \varphi)
\end{array}\right]^{T},
$$

$$
\sigma^{2}=\frac{N_{0}}{4 T_{c o h}}\left[\begin{array}{clrc}
R_{11}(0) & R_{12}(0) & 0 & 0 \\
R_{21}(0) & R_{22}(0) & 0 & 0 \\
0 & 0 & R_{11}(0) & R_{12}(0) \\
0 & 0 & R_{21}(0) & R_{22}(0)
\end{array}\right]^{T}
$$

where $R_{i j}$ is the autocorrelation $(i=j)$ or cross-correlation $(i \neq j)$ function of the local auxiliary signals, i.e., $R_{i j}=$ $E\left(L_{i} \otimes L_{j}\right)$. Here, we use Monte Carlo simulations to analyze the acquisition performance and assume that the Doppler frequency error and code delay error are small [10]. Meanwhile, the noise is generated using (20).

The detection probability of MBOC, sine-BOC $(2 n, n)$ and cosine-BOC $(2 \mathrm{n}, \mathrm{n})$ versus $\mathrm{CNR}$ with a fixed false alarm probability $P_{f a}=10^{-5}$ for different acquisition methods are shown in Fig. 7, Fig. 8 and Fig. 9, respectively. The test threshold can be asymptotically tuned from a straightforward evaluation of the Gaussian integral for fixed $P_{f a}$, under the $H_{0}$ hypothesis (i.e., absence of the tested code with the considered code offset) [17]:

$$
V=E\left[\left.Z_{k}(\tau)\right|_{H_{0}}\right]+\left(2 \cdot \operatorname{var}\left[\left.Z_{k}(\tau)\right|_{H_{0}}\right]\right)^{1 / 2} \cdot \operatorname{erf}^{-1}\left(1-2 P_{f a}\right)
$$

where

$$
Z_{k}(\tau)=\frac{1}{W} \sum_{w=1}^{W} \bar{R}(\tau)
$$

where $W$ is the accumulation blocks number of the power detector; $\bar{R}(\tau)$ is the synthesized correlation function of the proposed method.

In the simulation, the detection probability of traditional ambiguous acquisition is under the assumption that no false acquisition occurs and it is reviewed here for comparison. From Fig. 7, Fig. 8 and Fig. 9, it can be observed that with the increase of non-coherent summation number $N$ and the integral time $T_{c o h}$, the detection probability increase significantly. However, the non-coherent summations can widen the performance gap between the traditional acquisition scheme and the proposed method as shown in Fig. 8 and Fig. 9. The performance degradation is significant with a larger shape factor $k$ which is proportional to the weight coefficient $\alpha$. Since the weighted sum operation in (19) introduces more noise relative to the traditional method, the performance degradation seems to increase with the increasing of weight coefficient $\alpha$. However, it must be kept in mind that the comparison is based on the assumption that no false acquisition occurred for traditional acquisition scheme. From Fig. 7, it also can be seen that for MBOC signal, the proposed method outperforms the traditional acquisition scheme in terms of detection probability when the non-coherent summation number is low. That is mainly because the main peak of the SCF of the proposed method is the sum of two CCFs between the incoming signal and two local auxiliary signals. For MBOC with $k=0.1$, the signal to noise ratio (SNR) of the proposed method is slightly higher than the traditional method. However, with the increase of modulation order $M$ of BOC signals, the amplitude of the SCF peak decreases, resulting in degradation in detection performance; this degradation is more obvious 


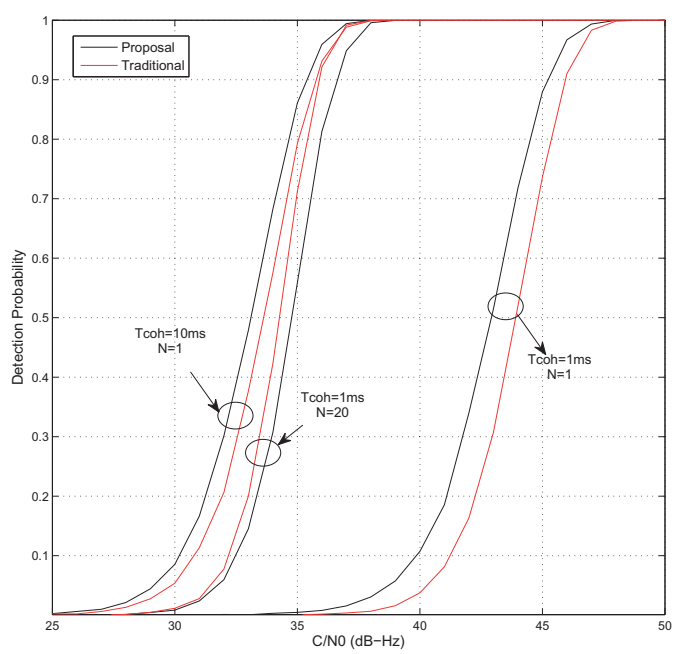

Fig. 7. The detection probability for MBOC, $P_{f a}=10^{-5}$, $k=0.1$.

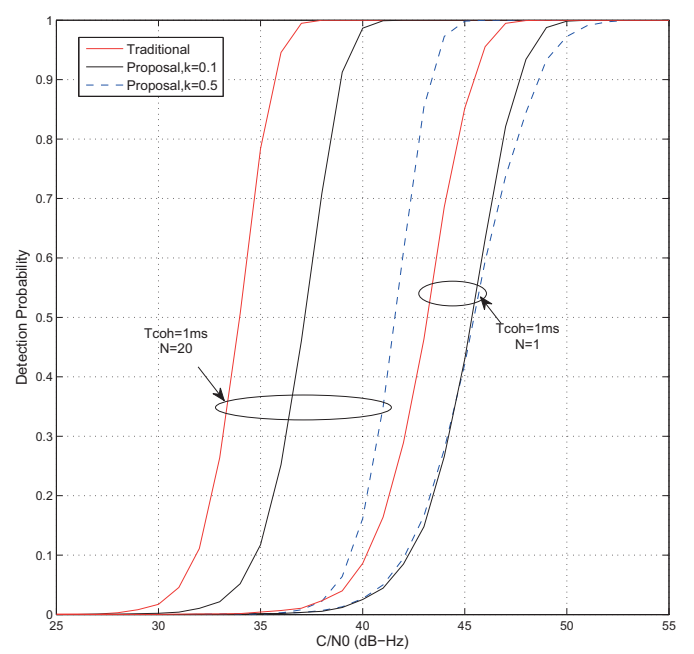

Fig. 8. The detection probability for sine-BOC(2n,n), $P_{f a}=$ $10^{-5}, k=0.1$ and $k=0.5$.

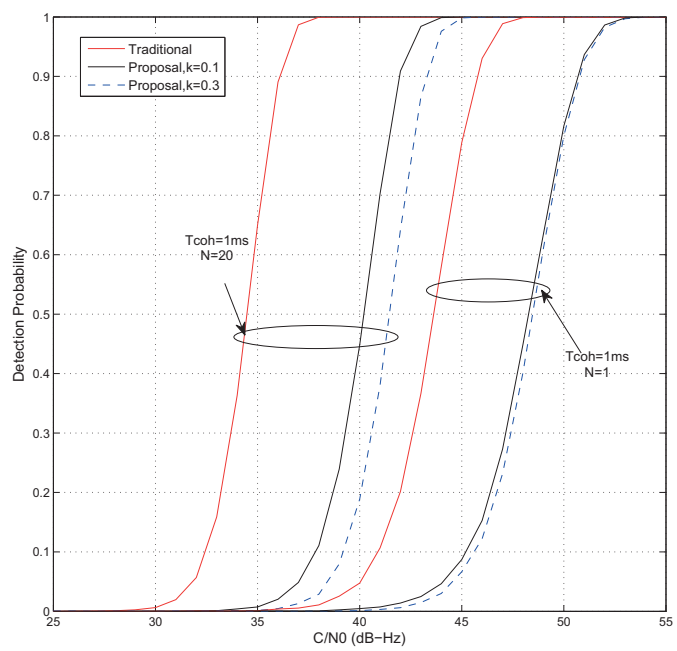

Fig. 9. The detection probability for cosine- $\operatorname{BOC}(2 \mathrm{n}, \mathrm{n}), P_{f a}=$ $10^{-5}, k=0.1$ and $k=0.3$. for cosine phased BOC signals comparing to Fig. 3, Fig. 4 and Fig. 5. However, it must be kept in mind that the comparison is based on the assumption that no false acquisition occurred. In practical applications, the multi-peak characteristic makes it easy for false detection to occur in the traditional acquisition scheme, especially in low $C / N_{0}$ environment. But the SCF of the proposed method does not contain any positive side peak, which makes the proposed technique more robust against false detection than traditional acquisition scheme. Meanwhile, considering that longer integral time leads to higher detection probability, the proposed method can be used for acquisition on data-less channels where the coherent integral time can be prolonged to compensate for its lack of detection performance.

It also can be seen form Fig. 3, Fig. 4 and Fig. 5 that the test criterion of the proposed method has a narrower peak comparing with BPSK-Like [18] [19] methods, which can provide sufficiently small code delay uncertainty. Therefore the additional transition from acquisition to tracking can be avoided. However, it also means that the proposed method needs smaller code delay bins which will increase mean acquisition time.

\section{Tracking Performance Analysis of the Proposed Method}

\subsection{S-curve Simulation of the Proposed Technique}

In order to evaluate the tracking performance, the Early-Minus-Late-Power (EMLP) discriminator is used hereafter. For the proposed method, the discriminator can be obtained as

$$
\begin{aligned}
D_{\text {proposal }}^{\text {ELMP }}=\left[\begin{array}{l}
{\left[\left(I_{B / L_{1}}^{L}+I_{B / L_{2}}^{L}\right)^{2}+\left(Q_{B / L_{1}}^{L}+Q_{B / L_{2}}^{L}\right)^{2}\right]} \\
-\left[\left(I_{B / L_{1}}^{E}+I_{B / L_{2}}^{E}\right)^{2}+\left(Q_{B / L_{1}}^{E}+Q_{B / L_{2}}^{E}\right)^{2}\right]
\end{array}\right] \\
-\alpha\left[\begin{array}{l}
\left.\left(I_{B / L_{1}}^{L}-I_{B / L_{2}}^{L}\right)^{2}+\left(Q_{B / L_{1}}^{L}-Q_{B / L_{2}}^{L}\right)^{2}\right] \\
-\left[\left(I_{B / L_{1}}^{E}-I_{B / L_{2}}^{E}\right)^{2}+\left(Q_{B / L_{1}}^{E}-Q_{B / L_{2}}^{E}\right)^{2}\right]
\end{array}\right]
\end{aligned}
$$

Figure 10 shows EMLP discriminator output of sine$\operatorname{BOC}(2 n, n)$ signal for the traditional [21-23], PCF [12] and proposed method without front-end filter and Early-Late spacing $d=0.1$ chips, respectively. It can be seen that the proposed method clearly removes any potential false lock points [16] [24]. Moreover, the discriminator stability domain is slightly larger than PCF and the traditional method: from $[-0.16 ; 0.16]$ chips for PCF and $[-0.15 ; 0.15]$ chips for the traditional method to $[-0.188 ; 0.188]$ chips for the proposed method. In addition, with the increase of shape factor $k$, the slope of the proposed method at $\tau \approx 0$ increased, but the discriminator stability domain remains unchanged. 


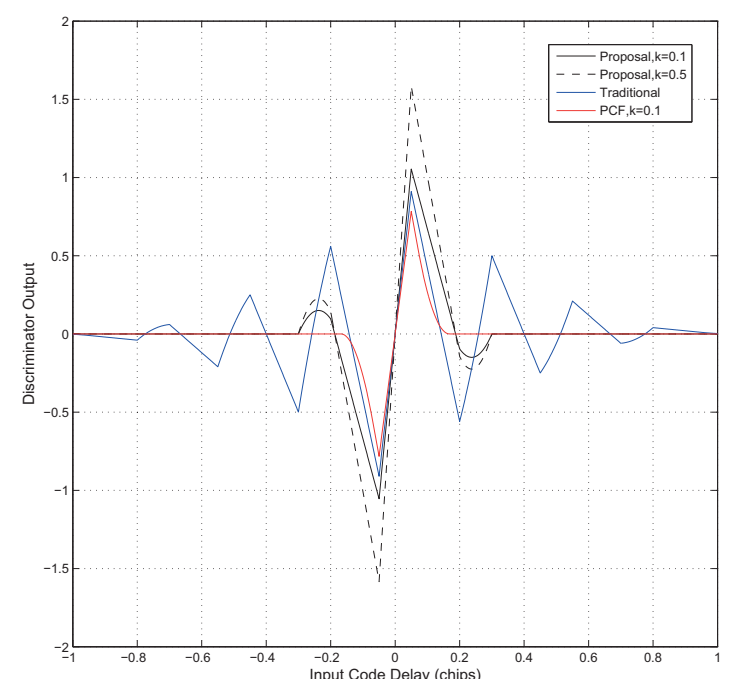

Fig. 10. S-curve of sine-BOC $(2 n, n)$ for traditional method, $P C F$ with $k=0.1$, and proposed method with $k=0.1$ and $k=0.5$, without front-end filter, and Early-Late spacing $d=0.1$ chips.

\subsection{Impact of Thermal Noise}

The BOC signals tracking accuracy depends on the thermal noise level, and the code tracking error standard deviation caused by thermal noise is defined as [25]

$$
\sigma_{\tau}=\frac{\sqrt{2 B_{L} T_{c o h}}}{G} \sigma_{D}
$$

where $\sigma_{D}$ is the measured standard deviation of the discriminator output, $B_{L}$ is the single-sided closed loop noise bandwidth in $\mathrm{Hz}, T_{\text {coh }}$ is the coherent integration time, $G=$ $\left.\frac{d D(\tau)}{d \tau}\right|_{\tau=0}$ is the normalized discriminator gain.

Figure 11 shows the standard deviation of code tracking error for different tracking technique with $B_{L}=2 \mathrm{~Hz}$, $T_{c o h}=1 \mathrm{~ms}, d=0.05$ chips. From Fig. 11, it can be seen that the proposed method has a worse performance than the traditional DLL and PCF $(k=0)$. However, there is only slight absolute gap between the proposed method and the traditional DLL with the CNR increasing. Although the tracking accuracy of the proposed method degraded comparing with the traditional DLL for BOC signals in the presence of thermal noise, it still outperforms the traditional DLL for BPSK signals. It also can be seen that the tracking accuracy curves of the proposed method for $k=0.1$ and $k=0.5$ nearly overlap, which means that the shape factor $k$ has no impact on the thermal noise caused code tracking error.

\subsection{Impact of Multipath}

To investigate the effect of multipath on the code tracking, in this paper, we consider a simple model of multipath with only one-path specular reflection, Meanwhile, we assume the amplitude and phase delay of the multipath are time-invariant over the time period of interest.

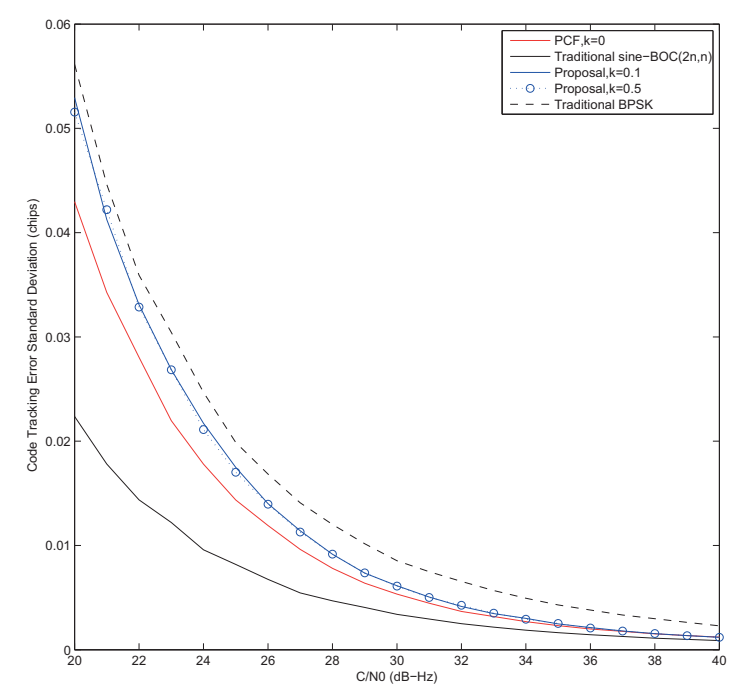

Fig. 11. Code tracking error standard deviation versus $C / N_{0}$ with $B_{L}=2 \mathrm{~Hz}, T_{c o h}=1 \mathrm{~ms}, d=0.05$ chips for sine$\mathrm{BOC}(2 \mathrm{n}, \mathrm{n})$ signal.

Figure 12 and Figure 13 show the multipath-induced error envelop for the traditional MBOC and BPSK tracking method, as well as for the proposed method with $k=0.1$ and $k=0.5$, respectively. In this simulation, the reflection path is $6 \mathrm{~dB}$ weaker than the direct path, and the Early-Late spacing, $d$, is 0.1 chips, and the frond-end bandwidth is infinite. Figure 14 and Figure 15 show the case for sine-BOC $(2 n, n)$ and cosine-BOC $(2 n, n)$ respectively with infinite front-end bandwidth, $d=0.1$ chips, and several values of $k$.

As shown in Fig. 12 and Fig. 13, the proposed method exhibits inferior multipath resistance performance in short and medium delay for MBOC signals. For delay $[0.08 ; 0.51]$ chips, the proposed method has inferior performance than traditional BPSK signal. But for delay [0.56;0.85] chips, it is superior to the traditional DLL for MBOC signals. For medium and long delay multipath, it exhibits familiar multipath mitigation performance with the traditional DLL for MBOC signals. However, it must be kept in mind that the proposed method completely removes ambiguity threat for both MBOC signals tracking and acquisition process.

From Fig. 14 and Fig. 15, it can be seen that, compared with the traditional DLL, the multipath mitigation performance of proposed method decreased slightly for sine$\operatorname{BOC}(2 n, n)$ and cosine-BOC $(2 n, n)$ signals. There is only slight absolute gap between the proposed method and the traditional DLL for cosine-BOC $(2 n, n)$ signal with the multipath delay increasing. But the proposed method outperforms the traditional DLL for BPSK signals significantly, especially for long delay multipath.

It should be noted that the multipath induced error envelops of the proposed method for $k=0.1$ and $k=0.5$ are nearly overlap as shown from Fig. 12 to Fig. 15, which means the changing of shape factor $k$ cannot improve the multipath resistance performance. The conclusion that the 


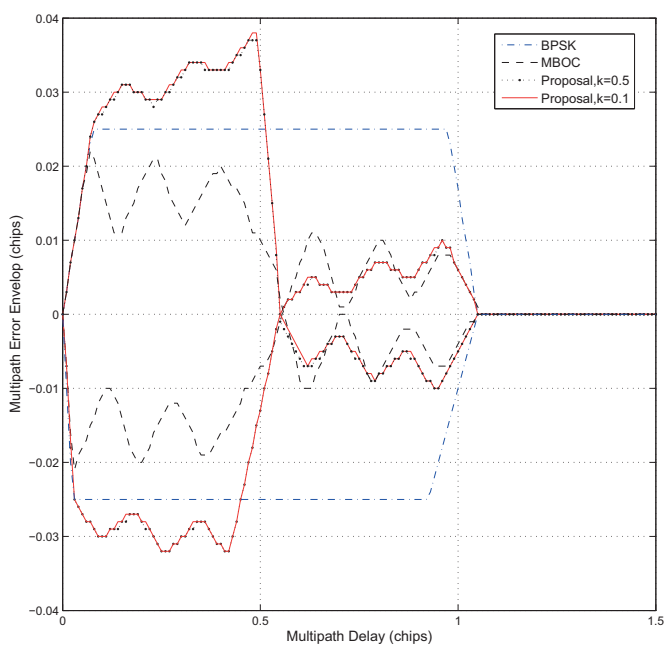

Fig. 12. Code tracking multipath envelop for MBOC, BPSK, and proposed method $(k=0.1$ and $k=0.5)$ for EarlyLate spacing of 0.1 chips, and infinite front-end filter.

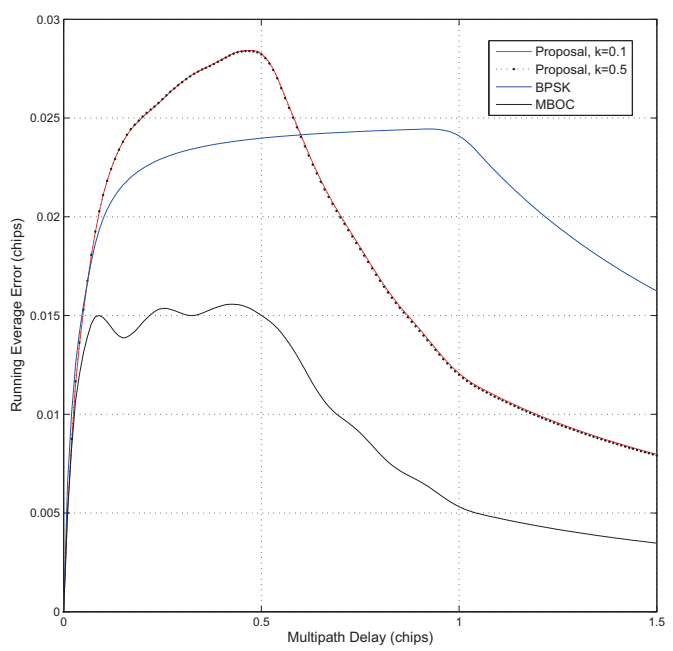

Fig. 13. Running average errors of BPSK, $M B O C$ and proposed method ( $k=0.1$ and $k=0.5)$, Early-Late spacing of 0.1 chips, and infinite front-end filter.

shape factor $k$ has no influence on multipath resistance is similar with the thermal noise resistance performance mentioned above. However, a lower value of $k$ remains a relative higher detection probability for the proposed method. Therefore, a lower $k$, such as $k=0.1$, is suggested in the practical applications.

\section{Implementation Issues}

In the proposed method, the local modulated symbols are step-shape and $k$ is a real tunable variable within $[0,1)$, so there are some difficulties to implementing the correlators and proposed modulators using logic circuits. To solve the problem, we herein reanalysis the SCF of the proposed method.

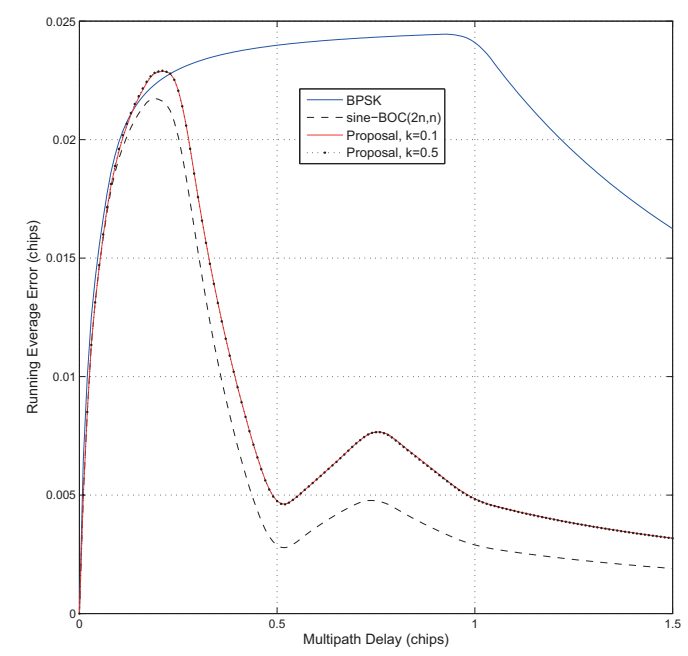

Fig. 14. Running average errors of BPSK, sine-BOC $(2 n, n)$ and proposed method ( $k=0.1$ and $k=0.5)$, Early-Late spacing of 0.1 chips, and infinite front-end filter.

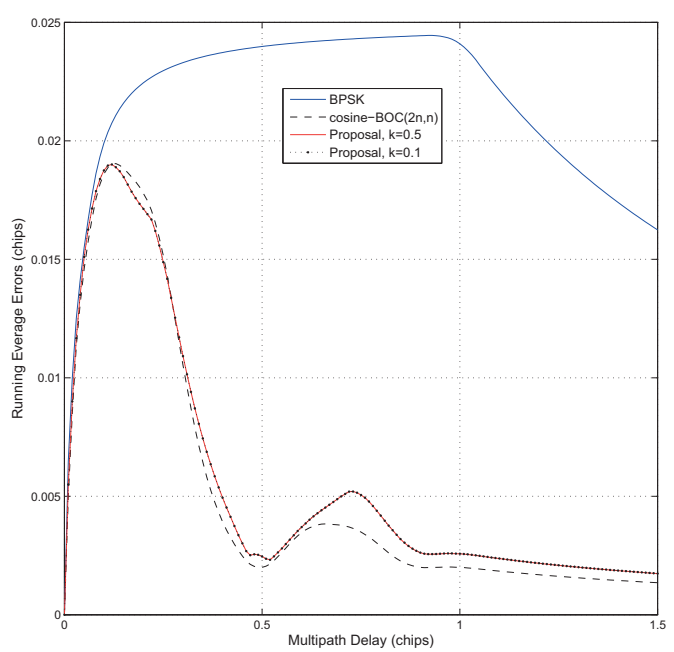

Fig. 15. Running average errors of BPSK, cosine-BOC $(2 n, n)$ and proposed method $(k=0.1$ and $k=0.5)$, Early-Late spacing of 0.1 chips, and infinite front-end filter.

In fact, the shape vector of local signal can be divided into two parts, i.e. $\mathbf{d}_{L}=\alpha \mathbf{d}_{L}^{(1)} \pm \beta \mathbf{d}_{L}^{(2)}$. Then the ACCF between BOC signal and local auxiliary signal can be expressed as

$$
\begin{aligned}
r_{k} & =\frac{1}{M} \sum_{i=0}^{M-1} d_{B O C, i} d_{L, k+i} \\
& =\frac{1}{M} \sum_{i=0}^{M-1} d_{B O C, i}\left(\alpha d_{L, k+i}^{(1)} \pm \beta d_{L, k+i}^{(2)}\right) \\
& =\alpha r_{k}^{(1)} \pm \beta r_{k}^{(2)}
\end{aligned}
$$

Note that the CCF between received BOC signal and local auxiliary signal is a linear combination of ACCF, therefore, after substituting (28) into the CCF, for example (6), then

$$
R_{B / L}(\tau)=\alpha R_{B / L}^{(1)}(\tau) \pm \beta R_{B / L}^{(2)}(\tau)
$$

where $R_{B / L}^{(1)}(\tau)$ and $R_{B / L}^{(2)}(\tau)$ are the CCFs between received BOC signal and local auxiliary signals with shape vectors 
$\mathbf{d}_{L}^{(1)}, \mathbf{d}_{L}^{(2)}$ respectively. In (11), the shape vectors of local auxiliary signal $d_{1}(k)$ and $d_{2}(k)$ can be rephrased as

$$
\begin{aligned}
& d_{1}(k)=d^{(1)}(k)+k d^{(2)}(k) \\
& d_{2}(k)=k d^{(1)}(k)+d^{(2)}(k)
\end{aligned}
$$

where

$$
\begin{aligned}
& d^{(1)}(k)=\left[\sqrt{\frac{2 M}{1+k^{2}}}, 0, \ldots, 0\right]^{T} \\
& d^{(2)}(k)=\left[0, \ldots, 0, \sqrt{\frac{2 M}{1+k^{2}}}\right]^{T}
\end{aligned}
$$

then

$$
\begin{gathered}
R_{B / L_{1}}(\tau)=R_{B / L}^{(1)}(\tau)+k R_{B / L}^{(2)}(\tau) \\
R_{B / L_{2}}(\tau)=k R_{B / L}^{(1)}(\tau)+R_{B / L}^{(2)}(\tau) .
\end{gathered}
$$

It can be proved that the shape of the $\mathrm{CCF}$ between received BOC signal and local auxiliary signal keeps unchanged. However, the spreading symbols in this form are easier to implement using logic circuits.

In this form, after the carrier stripping process, the received signal does not need to be correlated with two stepshape symbol modulated local signals. It just multiplies a replica of the spreading code and then does gating integral and dump. $R_{B / L_{1}}(\tau)$ and $R_{B / L_{2}}(\tau)$ can be obtained as a linear combination of one integrator output which only works during the first $T_{s}$ interval of each $T_{c}$ and another integrator output which only works during the last $T_{S}$ interval of each $T_{c}$. The integration time is $T$. Such a high speed but simple process is easily implemented in an ASIC or FPGA, and this process provides filtering and resampling.

Note that the number of correlators the proposed technique employs is the same as the bump-jumping method and $\mathrm{ASPeCT}$, which is small compared with those which require dozens of correlators to obtain an unambiguous discriminator S-curve, such as S-curve shaping technique proposed in [26].

\section{Conclusions}

In this paper, based on the combination of constitutive sub-correlations of the BOC autocorrelation, an unambiguous acquisition and tracking technique for generic BOC signal is proposed, which is suitable for MBOC, sine phased and cosine phased BOC signals. Theoretic analysis and simulation results show that the proposed method completely removes ambiguity problem in both BOC signals acquisition and tracking process.

In the acquisition process, for MBOC signals, the detection probability of the proposed method keeps higher than the traditional acquisition method if a low value of shape factor $k$ is adopted. Whereas for higher modulation order sine and cosine phased BOC signals, the acquisition sensitivity of the proposed method decreases with the increase of shape factor $k$, but the sensitivity degradation of the proposed method is acceptable when the shape factor $k$ keeps small. In the tracking process, the discriminator output of the proposed method keeps a slightly larger stability domain compared with the traditional tracking and PCF methods, which can maintain the DLL stability in low $C / N_{0}$ environment. In term of thermal noise resistance performance, the proposed method has very limited degradation compared with PCF and traditional tracking method with the $C / N_{0}$ increasing. The multipath rejection performance of the proposed method is decreased compared with the traditional tracking method, especially for MBOC signals, meanwhile, the degradation decreases with the increase of modulation order, especially for cosine phased BOC signals. It must be kept in mind that the comparison is based on the assumption that no false acquisition and tracking occurred for the traditional method. But in practical applications, the multi-peak characteristic makes the traditional acquisition and tracking schemes easy to acquire or track false peak.

Concerning the shape factor $k$, it is deserved to note that the shape factor $k$ has an obvious effect on BOC signals acquisition process but little impact on signals tracking accuracy. A large value of $k$ will amplify the noise, resulting in decreased detection probability. Therefore, a low value of $k$ is suggested to be chosen if the proposed method is used in the receiver. In addition, the proposed method is practicable and requires the same number of correlators as ASPeCT and the bump-jumping method. Moreover, it has an equivalent form to the proposed method whose correlators and modulators are easily implemented via logic circuits.

\section{Acknowledgments}

This work was supported by National Nature Science Foundation of China under grants 61102107 and 61374208, and by the Fundamental Research Funds for the Central Universities under grants HEUCFX41310.

\section{References}

[1] GODET, J., DE MATEO, J. C., ERHARD, P., et al. Assessing the radio frequency compatibility between GPS and Galileo. In Proceeding of U.S. Institute of Navigation GPS Conference. Portland (OR, USA), 2002, p. 1260-1269.

[2] BETZ, J. W. Binary offset carrier modulations for radionavigation. Navigation, Journal of the Institute of Navigation, 2002, vol. 48, no. 4, p. 227-246. DOI: 10.1002/j.2161-4296.2001.tb00247.x

[3] MARTin, N., LEBlond, V., GUIllotel, G., HEIRIES, V. BOC $(x, y)$ signal acquisition technique and performances. In Proceedings of the 16th International Technical Meeting of the Satellite Division of the Institute of Navigation (ION GPS/GNSS). Portland (OR, USA), 2003, p. 188-198.

[4] FISHMAN, P., BETZ, J. W. Predicting performance of direct acquisition for the M code signal. In Proceedings of the 2000 National Technical Meeting of The Institute of Navigation. Anaheim (CA, USA), 2000, p. 574-582.

[5] MAO, W.-L., HWANG., C.-S., HUNG, C.-W., et al. Unambiguous BPSK-like CSC method for Galileo acquisition. In 18th International Conference on Methods and Models in Automation and Robotics (MMAR). Miedzyzdroje (Poland), 2013, p. 627-632. DOI: 10.1109/MMAR.2013.6669983 
[6] BENEDETTO, F., GIUNTA, G., LOHAN, E. S., RENFORS, M. A fast unambiguous acquisition algorithm for BOC-modulated signals. IEEE Transactions on Vehicular Technology, 2013, vol. 62, no. 3, p. 1350-1355. DOI: 10.1109/TVT.2012.2228681

[7] FINE, P., WILSON, W. Tracking algorithm for GPS offset carrier signals. In Proceedings of the 1999 National Technical Meeting of The Institute of Navigation. San Diego (CA, USA), 1999, p. 671-676.

[8] HODGART, M., BLUNT, P., UNWIN, M. Double estimator - a new receiver principle for tracking BOC signals. Inside GNSS, Spring 2008, p. 26-36.

[9] YAO, Z., LU, M., FENG, Z. Unambiguous sine-phased binary offset carrier modulated signal acquisition technique. IEEE Transactions on Wireless Communications, 2010, vol. 9, no. 2, p. 577-580. DOI: 10.1109/TWC.2010.02.091066

[10] ZHOU, Y. Ambiguity mitigating technique for cosine-phased binary offset carrier signal. IEEE Transactions on Wireless Communications, 2012, vol. 46, no. 4, p. 1981-1984. DOI: 10.1109/TWC.2012.041612.110531

[11] KIM, H., LEE, Y., YOON, S. An unambiguous acquisition scheme for binary offset carrier signals. In 2013 International Symposium on Intelligent Signal Processing and Communications Systems (ISPACS). Naha (Japan), 2013, p. 340-344. DOI: 10.1109/ISPACS.2013.6704573

[12] YAO, Z., et al. Pseudo-correlation function-based unambiguous tracking technique for sine-BOC signals. IEEE Transactions on Aerospace and Electronic Systems, 2010, vol. 46, no. 4, p. 1782-1796. DOI: 10.1109/TAES.2010.5595594

[13] XU, D., LIU, M., SHEN, F. Ambiguity mitigating technique for multiplexed binary offset carrier signal tracking. IEEE Cоттиnications Letters, 2013, vol. 17, no. 11, p. 2021-2024. DOI: 10.1109/LCOMM.2013.091113.131685

[14] WARD, P. W. A design technique to remove the correlation ambiguity in binary offset carrier (BOC) spread spectrum signals. In Proceedings of the 59th Annual Meeting of The Institute of Navigation and CIGTF 22nd Guidance Test Symposium. Albuquerque (NM, USA), 2003, p. 146-155.

[15] PHAM-VIET, H., DAO-NGOC, C., NGUYEN-VAN, K. A scheme of cosine-BOC $(\mathrm{n}, \mathrm{n})$ side peaks cancellation for navigation applications. In 2013 International Conference on Advanced Technologies for Communications (ATC'13). Ho Chi Minh City (Vietnam), 2013, p. 180-184. DOI: 10.1109/ATC.2013.6698101

[16] JULIEN, O., MACABIAU, C., CANNON, M. E., LACHAPELLE, G. ASPeCT: unambiguous sine-BOC $(n, n)$ acquisition/tracking technique for navigation applications. IEEE Transactions on Aerospace and Electronic Systems, 2007, vol. 43, no. 1, p. 150-162. DOI: 10.1109/TAES.2007.357123

[17] BENEDETTO, F., GIUNTA, G. A self-synchronizing method for asynchronous code acquisition in band-limited spread spectrum communications. IEEE Transactions on Communications, 2006, vol. 57, no. 8, p. 2410-2419. DOI: 10.1109/TCOMM.2008.08.070030

[18] HEIRISES, V., ROVIRAS, D., RIES, L. Analysis of non-ambiguous BOC signal acquisition performance. In Proceedings of the 17th International Technical Meeting of the Satellite Division of The Institute of Navigation (ION GNSS). Long Beach (CA, USA), 2004, p. 671-676.

[19] LOHAN, E. S., BURIAN, A., RENFORS, M. Low-complexity unambiguous acquisition methods for BOC modulated CDMA signals. International Journal of Satellite Communications and Networking, 2008, vol. 26, p. 503-522. DOI: 10.1002/sat.922

[20] HOLMES, J. K. Coherent Spread Spectrum Communications. Melbourne (FL, USA): Krieger Publishing Co., 1990. ISBN: 9780071382151

[21] VAN DIERENDONCK, A. J. GPS receivers. In Global Positioning System: Theory and Applications. New York (USA): American Institute of Astronautics and Aeronautics, 1996, p. 329-408. ISBN: 978-1563471063
[22] WARD, P. Satellite signal acquisition and tracking. In Understanding GPS-Principles and applications. Norwood (MA, USA): Artech House, 1997, p. 119-218. ISBN: 978-1580538947

[23] HOLMES, J. K., RAGHAVAN, S. H., LAZAR, S. Acquisition and tracking performance of NRZ and square-wave modulated symbols for use in GPS. In Proceedings of the 54th Annual Meeting of The Institute of Navigation. Denver (CO, USA), 1998, p. 611-626.

[24] JULIEN, O., MACABIAU, C., CANNON, M. E., et al. New unambiguous BOC $(\mathrm{n}, \mathrm{n})$ tracking technique. In Proceeding of NaviTec Conference. Noordwidjk (The Netherlands), 2004, 8 pages.

[25] NUNES, F. D., SOUSA, F. M. G., LEITAO, J. M. N. Gating functions for multipath mitigation in GNSS BOC signals. IEEE Transactions on Aerospace and Electronic Systems, 2007, vol. 43, no. 3, p. 951-964. DOI: 10.1109/TAES.2007.4383585

[26] PANY, T., IRSIGLER, M., EISSFELLER, B. S-curve shaping: A new method for optimum discriminator based code multipath mitigation. In Proceedings of the 18th International Technical Meeting of the Satellite Division of The Institute of Navigation (ION GNSS). Long Beach (CA, USA), 2005, p. 2139-2154.

\section{About the Authors ...}

Feng SHEN was born in Jiangsu,China in 1981. He received his Ph.D. from Harbin Engineering University in 2009. He is now an associate professor at the College of Automation, Harbin Engineering University, Harbin, China. He developed the signal acquisition and tracking of the Spread spectrum receiver for the GPS ground-based augmentation systems. From 2014 to 2015, he is a visiting academic with the School of Electrical Engineering, University of New South Wales. His research interests include weak signal acquisition and tracking, GNSS/INS integrated navigation system.

Guanghui XU (corresponding author) was born in Shandong, China in 1987. He received his B.S. from Weifang University, Shandong, China in 2011. He is currently working toward the Ph.D. degree at Harbin Engineering University. His research interests include satellite navigation, signal structure design and signal processing.

Joon Wayn CHEONG is a research associate at the School of Electrical Engineering, University of New South Wales (UNSW). He received his Ph.D. (2012) and B.E. (2008) from UNSW. He developed the firmware for the space-qualified Namuru family of GPS/GALILEO integrated receivers under the Garada, Biarri and QB50 project, where he cracked the Locata pseudolite positioning system's code and derived a hybrid Collective Detection algorithm. His other research interests in the GNSS field include weak signal acquisition, A-GPS, GNSS/pseudolite integrated signal processing, GNSS interference and spoofing. He authored more than 15 peer-reviewed publications.

Haiyu FENG was born in Shandong, China in 1989. She received her B.S. from Binhai University, Shandong, China in 2011. She is currently working toward the M.Sc. degree at Harbin Engineering University. Her research interests include satellite navigation, target detection under sea clutter. 